\title{
Análisis de Vídeo-lecciones en MOOC enfocados en la formación pedagógica de docentes en Educación Superior. Un estudio de caso
}

\author{
Edna Manotas Salcedo. Universidad del Norte. Colombia \\ Amor Pérez Rodríguez. Universidad de Huelva. España \\ Paloma Contreras Pulido. Universidad de Huelva. España
}

Recepción: 06.08.2018 | Aceptado: 15.09.2018

Correspondencia a través de ORCID: Edna Manotas Salcedo

iD $0000-0002-4080-2740$

Citar: Manotas Salcedo, E., Pérez Rodríguez, A. y Contreras Pulido, P. (2018). Análisis de Vídeo-lecciones en MOOC enfocados en la formación pedagógica de docentes en Educación Superior. Un estudio de caso. ReiDoCrea, 7, 248-259.

\begin{abstract}
Resumen: El objetivo de esta investigación es analizar un grupo de vídeo-lecciones contenidas en Cursos Masivos en Línea (MOOC) (por sus siglas en inglés) enfocados en la formación pedagógica de docentes de Educación Superior. Método: Se realizó el análisis de contenido de 104 vídeo-lecciones en 3 cursos publicados en la plataforma Miriada X. El proceso tuvo dos fases, una, en la que se diseñó e implementó un instrumento para caracterizar el inicio, el desarrollo y el cierre de las vídeo-lecciones como unidad de comunicación, y otra, que consistió en la organización, transcripción y codificación de las vídeolecciones mediante el criterio de cronología, que permitió determinar tendencias y patrones. Resultados: Se evidenció un uso básico de herramientas audiovisuales y la tendencia a diseñar clases netamente expositivas detonando ausencia de elementos de storytelling. Conclusiones: Estos resultados son relevantes para el diseño instruccional de recursos audiovisuales para cursos masivos y para configurar propuestas creativas en el diseño de ambientes virtuales de aprendizaje, especialmente enfocados en la formación docente.
\end{abstract}

Palabras clave. MOOC | Vídeos Educativos

Analysis of Video-lessons in MOOC focused on the pedagogical training of teachers in Higher Education. A case study

Abstract: The purpose of this research is analyzing a group of video-lectures in Online Massive Courses (MOOC), which are focused on the pedagogical development of Higher Education faculty. Method: The content analysis of one hundred and four video-lectures contained in three courses of Miriada $\mathrm{X}$ was made. This process had two phases, the first one was to design and implement an instrument to characterize the beginning, progress and closure of the video-lectures as a communication unit and, the second one consisted in the organization, transcription and codification of these video-lectures to determine trends and patterns. Results: It was evidenced a basic use of audiovisual tools and the tendency to design clearly expository classes, showing absence of elements of storytelling. Conclusions: These results are relevant to the instructional design of audiovisual resources for massive courses and to configure creative proposals for the design of online learning environments, especially focused on faculty development.

Keywords: MOOC | Educational Videos

\section{INTRODUCCION}

En una clase presencial de más de 120 estudiantes, captar la atención de quien nos escucha es casi como cazar una liebre. El reto: que el discurso y las actividades de aprendizaje sean lo suficientemente interesantes para lograr cumplir con los resultados de aprendizaje planteados. Teniendo en cuenta que es un grupo extenso, debemos tomar decisiones sobre el diseño de cada clase de manera que pueda responder a esta necesidad. Este tipo de confrontaciones sobre lo personalizado y lo masivo, también ocurre en ambientes virtuales, especialmente con la aparición de los Cursos Masivos en Línea, MOOC - por sus siglas en inglés -. Se trata de contar con un 
diseño de curso lo suficientemente potente, basado en recursos digitales, para lograr establecer un ritmo de trabajo con cientos de estudiantes alrededor del mundo, dispares culturalmente y con distintos tipos de estilos de aprendizaje. Para el caso de los MOOC que están dirigidos a docentes, foco de esta investigación, se espera que presenten metodologías innovadoras que se conviertan en ejemplos o modelos a replicar. Al respecto, Ho, Chuang, Reich, Cody, Whitehill, Northcutt, Williams, Hansen, López y Petersen (2015) encontraron en una caracterización de la población que accede a un MOOC, que el $39 \%$ se identifica como antiguo o actual maestro. Es prioritario profundizar en el valor de los MOOC para fortalecer el desarrollo de competencias docentes. "La fuerte participación por parte de profesores sugiere que incluso los participantes que estén interesados en una certificación todavía pueden hacer un uso productivo de un MOOC". (Allen, 2015, p. 4).

En este sentido, el estudio se planteó: analizar e identificar la pertinencia didáctica de un grupo de vídeo-lecciones contenidas en cursos masivos en línea (MOOC) enfocados en la formación pedagógica de docentes de educación superior. Se analizó el caso de tres cursos de la plataforma en español Miriada X. En la parte inicial del artículo se demarcan los antecedentes sobre el tema y el marco conceptual alrededor de la formación virtual dirigida a docentes, diseño instruccional de MOOC y de vídeolecciones, luego se explica la metodología, los resultados y la discusión que presenta recomendaciones para la producción y diseño de recursos audiovisuales contenidos en cursos enfocados a docentes.

\section{Formación pedagógica en Educación Superior: ¿un saber especifico sin didáctica?}

Para que las universidades puedan cumplir su misión requiere de docentes que no sólo tengan conocimiento científico, sino didáctico que le apunten al aprendizaje significativo y para esto las mismas instituciones deben generar estrategias de formación (Cáceres, Lara, Iglesias, García, Bravo, Cañedo, Valdés, 2013).Las acciones educativas dirigidas a docentes deben potenciar su desarrollo profesional (Lowenthal, 2008; Montiel, 2009; González, 2005).

En este orden de ideas, el docente universitario cuenta con características especiales. Es un científico, en la mayoría de los casos, con estudios de doctorado y postdoctorado en temas especializados y que no necesariamente llega preparado para enfrentarse a la vida y dinámica del aula de clases. Este tipo de profesor requiere la combinación de tres elementos clave: conocimiento del contenido de su materia (por ejemplo, saber matemáticas), así como pedagógico (por ejemplo, saber enseñar matemáticas) incluso ser un experto en el tema, no lo convierte en un buen profesor (Gikandi, 2011; Lambert, 2012, Moscoso y Hernández, 2015; Shulman, 1986)

Con estos desafíos, no podemos olvidar que la docencia universitaria trae a colación la imagen de un experto que conoce mucho de una disciplina y tiene como función principal transferir y "dictar" a sus estudiantes ese conocimiento. Es casi que imperativo imaginarlos sólo preparando clases magistrales o lecturas. Martínez y De Castro (2013) concluyen, por ejemplo, que las tendencias actuales en pedagogía universitaria indican que el rol del docente no es transmitir conocimiento, sino crear ambientes y condiciones que conduzcan al estudiante a un aprendizaje profundo, duradero y significativo. Es decir, el docente pasa de ser transmisor de información a diseñador de espacios que ayuden a generar y facilitar aprendizajes.

Al respecto, Chehaybar (2006) concibe la formación docente como un proceso vinculado a las concepciones educativas, que es contextualizado por la situación socio-cultural de las regiones. Para este autor, la formación se vincula a la unión 
entre la administración y las políticas educativas, así como los avances pedagógicodidácticos que se reflejan en los currículos. Sin embargo, en su opinión, la formación del profesorado ha respondido en su mayor parte a las concepciones institucionales y los profesores de este nivel, cuentan con una especificidad temática y además deben cumplir labores de docencia, investigación y extensión.

Para Padilla, López y Rodríguez (2017) si bien, hay que cumplir con las políticas de cada institución, la formación docente hay que asumirla como un proceso continuo y flexible. Los aprendizajes, para el docente con estas características, se pueden generar en distintos contextos tales como la práctica profesional, las interacciones con colegas, la cursos de postgrado y sus vidas personales, entre otros escenarios (Bransford, Brown y Cocking, 2000).

De acuerdo con lo anterior, formar docentes en los niveles de educación superior supone un reto bastante mayúsculo. Estamos hablando de un grupo de profesores, que no ha sido formado sobre cómo se aprende, pero que es experto en un tema en particular. A la vez, los perfiles varían mucho en edad y niveles de formación académica. Adicionalmente, por las dinámicas del sistema, hay grupos de docentes contratados por horas de clase, que no son fáciles de agrupar en un mismo espacio, como sí suele suceder con docentes de educación básica y media. Estos factores, sumados a las particularidades de cada contexto, y el poco tiempo que suelen tener los docentes, hacen que el reto para formarlos en cómo diseñar ambientes de aprendizaje sea cada vez más complejo.

En distintas universidades a nivel mundial se han organizado centros de apoyo a la docencia en donde se ofrecen diplomados, cursos cortos, conferencias y actividades formativas. Hoy en día, entre los factores que impulsan el reciente interés en desarrollo profesional docente son: el uso de tecnología para enseñar y aprender; un cambio de enfoques pedagógicos hacia el constructivismo, el aprendizaje activo y un énfasis en la educación basada en competencias (Charalambos y Glass, 2007).

La agenda de formación docente en Educación Superior se está complejizando cada vez más por la creciente sofisticación del aprendizaje por el uso de las tecnologías, los procesos de acreditaciones institucionales y por el cambio en el alumnado: grupos más heterogéneos y con la atención dividida en redes sociales. Al mismo tiempo, los docentes, en muchos casos, no están obligados a participar de estas formaciones y su asistencia es baja por el creciente interés de las universidades en privilegiar la escritura y publicación de artículos científicos (Lee, 2010; Stevens, Dunlap, Bates, Lowenthal, Wray, y Switzer, 2005; Villalobos y Melo, 2008).

Muchas veces, los docentes reciben incentivos por publicar artículos en revistas indexadas, pero no por participar de programas de formación docente o realizar cambios significativos en su práctica de aula. Si los planes de formación son voluntarios, la participación tiende a la baja. En la mayoría de los casos, los docentes argumentan falta de tiempo para participar y se evidencia un escaso compromiso en poder lograr un equilibrio entre sus funciones. Este aspecto motivacional, depende también de la universidad, si, institucionalmente se hace énfasis en la investigación o la extensión, es natural que la parte docente no sea tomada en cuenta de manera masiva (Stevens, et al., (2005).

De este modo, para quienes organizan este tipo de cursos al interior de una facultad, lo más complicado es que de verdad, el docente, con todas estas dificultades en el contexto, cambie su enseñanza. Es decir, que se pueda impactar realmente en sus diseños de aula. Con toda esta problemática, los cursos para docentes en educación superior, tradicionalmente, han sido pensados desde las lógicas presenciales, sin embargo, con las oportunidades que abre la educación a distancia mediada por tecnología, se han abierto nuevas opciones sobre el tema. 


\section{Formación pedagógica a través de cursos en línea: el desafío por desaprender.}

Construir un ambiente virtual de aprendizaje implica adaptar herramientas tecnológicas para vencer las barreras comunicativas que expresa la distancia entre docentes y estudiantes. El entorno debe cumplir una función pedagógica, contar con la tecnología apropiada y tener los aspectos organizativos de gestión de comunidad (Merril, 2002; Salinas, 2004). Lograr todos estos componentes de manera efectiva toma tiempo y equipos de trabajo interdisplinarios, y si, el espacio virtual, está dirigido a la formación docente, supone una demanda mayor en cuanto a su diseño instruccional, modos de comunicación y enfoque pedagógico, por tanto, se busca que sirvan de modelo para una población que necesita cada vez más herramientas para innovar en su práctica de aula. Si de lo que se trata es de proveer un recurso de formación, que no vea al docente de educación superior, como un ente experto independiente que maneja una disciplina, se requiere de otras narrativas y diseños novedosos para las clases. Se requieren cuidadosos diseños contextualizados (Vivian, Falkner y Falkner (2014).

Al respecto, distintos estudios ofrecen un marco de referencia sobre la formación docente en línea (Charalambos, 2000; Literat, 2015; Rama, 2008) con componentes enmarcados desde el constructivismo, el cognitivismo y las comunidades de práctica. Distintas posturas conceptuales señalan que la enseñanza virtual para la formación de docentes debe centrarse en ayudar a los maestros a desaprender sus creencias, valores, suposiciones e inclusive su propia cultura sobre lo que entienden acerca de enseñar y aprender. Esto no puede hacerse de manera inmediata y requiere de procesos constantes, lentos y sostenidos en el tiempo. Este factor es una amenaza para la formación pedagógica en línea, ya que, generalmente, se esperan resultados muy rápidos (Dede, 2014). Sin embargo, el desarrollo de cursos en línea para la formación del profesorado aún está en etapa de maduración ya que se ha centrado en desarrollar habilidades para usar las tecnologías, pese a esto, es ideal porque ofrece flexibilidad términos de espacio y tiempo (Dabner, Davis, y Zaka, 2012; González, 2005; Lowenthal, 2008; Montiel, 2009).

En este sentido los MOOC se convierten en una opción, aunque son escasos los estudios sobre si este tipo de cursos, están impactando las prácticas de aula positivamente (Jobe, Östlund, y Svensson, 2014; Singh, 2014) pese a esto, los docentes ven los cursos masivos en línea como una oportunidad para ampliar sus redes de trabajo con colegas de distintas partes del mundo. Al respecto, la literatura señala como desventaja el riesgo de que se conviertan en salas de conferencia replicando modelos tradicionales de enseñanza (Silva y Salgado, 2014).

Al respecto, sobre la situación de los MOOC enfocados en este tema. Estudios previos señalan que una gran parte de los MOOC utiliza una metodología magistral y promueve estrategias de reproducción de contenido y esto no es distinto en los cursos diseñados para la formación de docentes (Calvo, Rodríguez y Fernández, 2016; Raposo, Martínez y Sarmiento, 2015). Esto puede concluirse analizando las clases que están cargándose en estos MOOC. La vídeo-lección resulta fundamental en el análisis (Chauhan y Goel, 2015; Rangel, 2007). Las vídeo-lecciones en la educación superior se encuentran insertas en una estructura fija enmarcadas en un diseño instruccional que no tiene muchas variaciones (Rose, 2009; Winslett, 2014). Generalmente estos cursos presentan una serie de pruebas y test después de la visualización de una vídeo-lección. Están basados en la adquisición de contenidos y se centran en un modelo de evaluación muy parecido a las clases tradicionales con unas pruebas estandarizadas (Lei, Yeung, Kwok, Lau y Ang, 2016; SCOPEO, 2013).

El uso de recursos tecnológicos en las prácticas docentes universitarias no siempre han representado una innovación pedagógica. La educación superior se ha apoyado en un modelo de enseñanza basado en las clases magistrales, en la lectura y 
memorización de textos (Area, 2000). El desarrollo profesoral no es estático y para cambiar viejas prácticas no sólo se trata de subir cursos a plataformas (Lovegrove, Mackness, Roberts y Waite, 2013; Monney (2010)

\section{Métodos: participantes, instrumentos, procedimiento y análisis de datos.}

Este es un estudio de caso y se centró en un análisis de contenido, una técnica de investigación para hacer inferencias válidas y replicables a partir de textos (u otro significado significativo) en los contextos de su uso (Krippendorff, 2013, p. 24). De igual manera, Igartua (2006) identifica que la funcionalidad del análisis de contenido para estudios de comunicación está representada en su utilidad para describir las características de los mensajes de forma univariada así como también, para identificar las relaciones entre las distintas características de los mismos. Se tomaron cursos de la plataforma Miriada $X$, una de las más relevantes en español y con más de 20 mil suscriptores. Esta muestra fue determinada mediante la técnica "muestreo intencional no probabilístico". Hernández, Fernández y Baptista (2010), afirman: "En las muestras no probabilísticas, la elección de los elementos no depende de la probabilidad, sino de causas relacionadas con las características de la investigación o de quien hace la muestra" (p. 190) Para escoger los cursos, se realizó una búsqueda general en la plataforma escogida, por las palabras: "pedagogía", "educación superior", "docencia", "docencia universitaria", "formación docente". Se identificaron 28 cursos que cumplían con este criterio. El Luego, se seleccionaron los cursos teniendo en cuenta los siguientes criterios de inclusión:

- Que el curso contará con un grupo de más de 20 vídeo-lecciones para analizar

- Que estuviera dirigido a docentes de Educación Superior

- Que estuvieran activos (publicados) o con los contenidos disponibles para consulta a la fecha del análisis.

- Que el curso estuviera publicado en castellano

\section{Criterios de exclusión:}

- Que estuviera centrado en documentos, texto escrito u PDF

- Que estuvieran dirigidos a educación básica primaria

- Que estuvieran en otro idioma, distinto al español

Con estos criterios se identificaron tres cursos que cumplían con todos estos ítems al corte de la investigación. La muestra total estuvo conformada por 104 vídeo-lecciones con una duración estándar entre los 6 y los 12 minutos.

\begin{tabular}{|l|l|l|}
\hline Tabla 1. Título de los cursos, universidad que lo ofrece y número de vídeo-lecciones \\
\hline Título del curso & Universidad & $\begin{array}{l}\text { Número de vídeo- } \\
\text { lecciones }\end{array}$ \\
\hline Ética en la investigación universitaria & Universidad de País Vasco & 40 \\
\hline $\begin{array}{l}\text { Pasos básicos para un aprendizaje personalizado en } \\
\text { el aula }\end{array}$ & $\begin{array}{l}\text { Universidad Politécnica de } \\
\text { Madrid }\end{array}$ & 39 \\
\hline $\begin{array}{l}\text { Construcción de un Curso Virtual en la Plataforma } \\
\text { Moodle }\end{array}$ & $\begin{array}{l}\text { Universidad San Martín de } \\
\text { Porres }\end{array}$ & 25 \\
\hline
\end{tabular}

Fuente: Elaboración propia 


\section{Técnicas para la recogida de datos}

La recolección de datos se hizo en dos momentos. El primero se hizo a través de un instrumento de 25 items, el cual fue sometido a juicio de expertos y se puede consultar en el siguiente link: https://videoleccionesmooc.questionpro.com/ Los temas clave que aborda este instrumento están centrados en caracterizar el inicio, el desarrollo y el cierre de las vídeo-lecciones, el uso de recursos audiovisuales y de la utilización de principios de Storytelling. El segundo proceso consistió en la organización y transcripción de las vídeo-lecciones mediante el criterio de cronología (Sampieri, Fernández y Baptista, 2014), atendiendo al orden en que fueron colgados en el curso virtual en la plataforma y en sus copias en Youtube. Se realizó codificación abierta que consistió en analizar las vídeo-lecciones y determinar categorías. Se recorrió cada video-lección y se marcaron las categorías con colores. Las técnicas para generar las categorías fueron las de escrutinio tales como la identificación de repeticiones, conceptos locales, similitudes y diferencias entre las video-lecciones en el contenido y lenguaje utilizado. Para identificar las relaciones entre estas categorías, se utilizaron los mapas conceptuales y esquemas con el fin de encontrar cómo se vinculan los conceptos entre las vídeo lecciones y el curso virtual en general para determinar patrones. Seguidamente se procedió con la interpretación de los datos y relacionando los datos obtenidos en ambas fases. Cabe resaltar que durante todo el proceso se realizó lo que se denomina una bitácora para ir llevando el detalle del análisis.

En la tabla 2, se puede observar un ejemplo de las transcripciones realizadas.

Tabla 2. Organización del contenido. Vídeo-lecciones. Curso 1.

\begin{tabular}{|c|c|c|}
\hline $\begin{array}{c}\text { VIDEO DE } \\
\text { PRESENTACIÓN. }\end{array}$ & $\begin{array}{c}\text { INTRODUCCIÓN A LA } \\
\text { PLATAFORMA EDUCATIVA } \\
\text { MOODLE }\end{array}$ & $\begin{array}{l}\text { EL AULA VIRTUAL Y } \\
\text { SU EDICIÓN. }\end{array}$ \\
\hline $\begin{array}{l}\text { Les doy a más cordial } \\
\text { bienvenida. Soy la } \\
\text { ingeniera Rosa Mori y } \\
\text { desde hace } 18 \text { años soy } \\
\text { parte del equipo de la } \\
\text { Universidad de San Martín } \\
\text { de Porres. Aquí trabajo } \\
\text { directamente en proyecto } \\
\text { de E-Learning y desde } \\
\text { aproximadamente } 9 \text { años } \\
\text { tengo experiencia como } \\
\text { docente virtual. }\end{array}$ & $\begin{array}{l}\text { Esta lección tiene por objetivo que el } \\
\text { participante reconozca la plataforma } \\
\text { Moodle como un espacio generador } \\
\text { de actividades educativas, así como } \\
\text { su utilidad y potencialidad en el } \\
\text { ámbito académico. }\end{array}$ & $\begin{array}{l}\text { Esta lección permite que } \\
\text { el participante reconozca } \\
\text { y construya su curso bajo } \\
\text { una estructura de aula } \\
\text { virtual y soporte } \\
\text { educativo apropiado para } \\
\text { generar r óptimos } \\
\text { aprendizajes. }\end{array}$ \\
\hline
\end{tabular}

Fuente: elaboración propia

En el siguiente gráfico se observa ejemplo del uso de mapas conceptuales para analizar las conexiones del contenido: 
Gráfico 1. Ejemplo de mapa conceptual elaborado

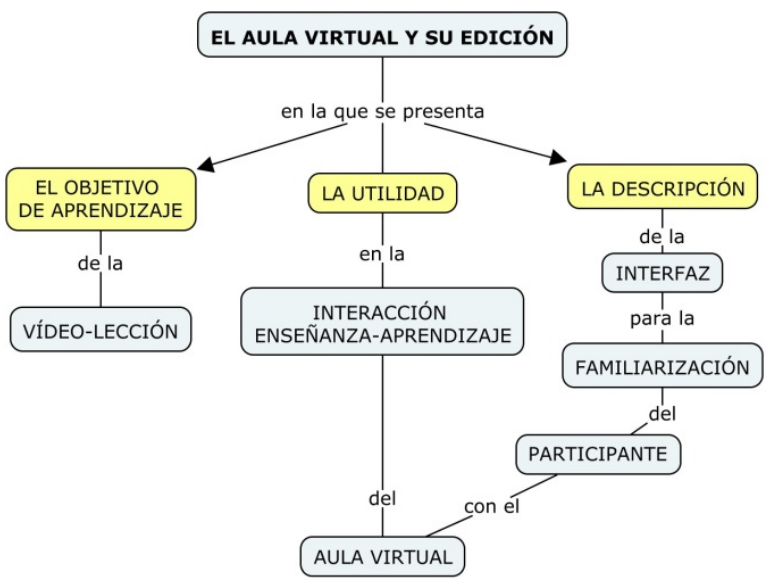

Fuente: Elaboración propia

\section{Resultados:}

En este apartado se describirán los hallazgos más relevantes de los dos procesos realizados.

En la totalidad de las vídeo-lecciones, se evidencia un claro dominio del tema a tratar. Esto se puede revelar en la manera en que los docentes explican el tema a la audiencia de manera secuencial. En la mayoría de los casos, el docente desglosa de manera detallada el tema, hace secuencias lógicas y enuncia el objetivo de aprendizaje de la vídeo-lección. Desde el punto de vista del contenido, se cumple con la consigna de informar sobre un tema en particular de manera ordenada. Esto se hace más evidente en la ausencia de recursos audiovisuales que puedan enriquecer el relato y ayudar al estudiante a completar funciones básicas dentro del proceso de pensamiento como agrupar y/o recordar. Básicamente el contenido podría volcarse a un podcast o archivo de audio sin ninguna diferencia. El tipo de formato más utilizado para la grabación es el Chroma donde se repite la presencia del profesor con un power point de fondo.

En cuanto a las partes de la clase: Inicio, desarrollo y cierre, se encontró una secuencia didáctica clásica en cuantos elementos de narrativa. Por ejemplo, en los inicios de las clases el discurso no cuenta con principios de engagement como frases llamativas, preguntas, contextualización, revisión de conocimientos previos de la clase anterior, entre otros recursos narrativos que permiten al estudiante mejorar su proceso de aprendizaje.

Esto demuestra, que algunas universidades hacen grandes esfuerzos en montar este tipo de recursos, pero no invierten tiempo en el desarrollo de guiones para organizar la clase en secuencias con principios de engagement claros y llamativos para la audiencia. También se observa, aunque en un porcentaje bajo, que algunos contenidos se enlazan con redes sociales y los estudiantes eventualmente pueden hacer aportes al contenido mismo. Este es un hallazgo interesante, toda vez, que la interacción con recursos variados cambia la forma en que el estudiante recibe el mensaje y le entrega nuevas coordenadas para su auto-aprendizaje. 
En cuanto al desarrollo y cierre de las clases, se encontró que la narración se concentra en temas disciplinares descuidando aspectos de narrativa como relacionar anécdotas personales, casos de la vida real y relacionando el tema con eventos que ayuden a situar al estudiante en cómo usar el contenido al que se está exponiendo. En cuanto a la parte técnica se evidenció un uso muy básico de recursos audiovisuales. No se evidencia el uso de infografías o animaciones. Se encontró también que no se plantean cambios de plano en el encuadre de la cámara y mucho menos giros en el ritmo de la narración de los profesores lo que hace que la clase decaiga en su estructura como unidad de comunicación potente. Es decir, las vídeo-lecciones tienen un sentido sobre qué contar, pero, en los tres cursos estudiados, la gran pregunta es cómo contar estas historias de manera que causen en la audiencia gran impacto y permitan estar mucho tiempo conectado con lo que se está diciendo.

Otro aspecto en el que coinciden es en la ausencia de un diseño instruccional para los vídeos. Es decir, el vídeo no cuenta con un diseño diferente según los temas, sino que obedece a estructuras fijas (power point, voz del profesor) lo que genera la oportunidad de plantear diseños instruccionales sólo para el material audiovisual.

Gráfico 2. Ejemplo de uso del power point.

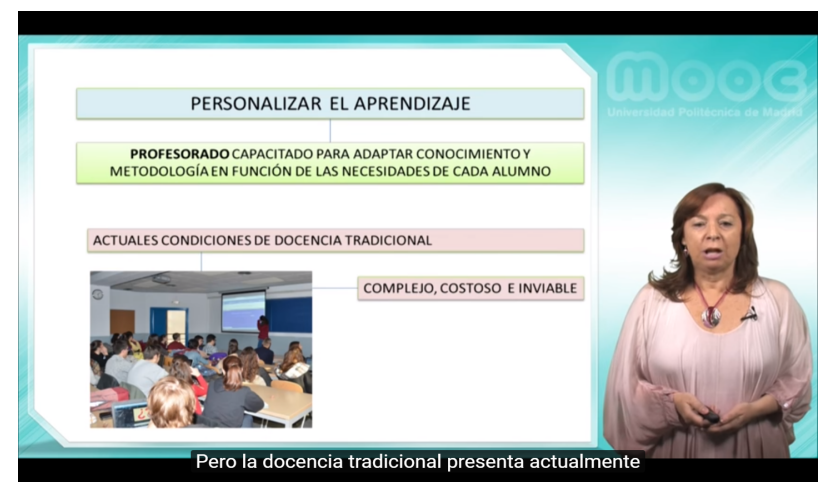

Fuente: Pantallazo del curso en Miriada X

Como se observa en la imagen predominan los usos clásicos del Power Point. En la mayoría de los casos, el docente no lo usa para enfatizar su discurso, sino que se muestra de manera aislada. Esto se evidencia, por ejemplo en la ausencia de conectores en el discurso sobre los gráficos. Es escaso el uso de palabras alusivas a los gráficos como "Aquí vemos que", "como pueden ver en:".

En cuanto al diseño de las vídeo-lecciones vs los resultados de aprendizaje que se plantean, en la siguiente tabla se explica:

Tabla 3. Caso 1. Relación de los objetivos del curso con el diseño de las vídeo-lecciones y la transferencia a la práctica docente.

\begin{tabular}{|l|l|l|l|}
\hline Curso & Objetivo del curso. & $\begin{array}{l}\text { Diseño de las vídeo- } \\
\text { lecciones }\end{array}$ & $\begin{array}{l}\text { Transferencia a la práctica } \\
\text { docente }\end{array}$ \\
\hline $\begin{array}{l}\text { Construcción de } \\
\text { un Curso Virtual en } \\
\text { la Plataforma } \\
\text { Moodle }\end{array}$ & $\begin{array}{l}\text { Reconocer la plataforma } \\
\text { Moodle como un espacio } \\
\text { generador de actividades y } \\
\text { potencialidad en el ámbito } \\
\text { académico }\end{array}$ & $\begin{array}{l}\text { El curso es de carácter } \\
\text { procedimental, por lo que las } \\
\text { lecciones son una serie de } \\
\text { tutoriales donde se explica } \\
\text { cada herramienta de Moodle. }\end{array}$ & $\begin{array}{l}\text { No se evidencia ningún tipo de } \\
\text { recurso para la transferencia a la } \\
\text { práctica de aula. }\end{array}$ \\
$\begin{array}{l}\text { Lecciones como "añadir un archivo", } \\
\text { "añadir una etiqueta", "abrir un } \\
\text { chat", "abrir un foro", "añadir un URL } \\
\text { son temáticas que están ilustradas } \\
\text { en muchos tutoriales disponibles. }\end{array}$ \\
\hline
\end{tabular}


Tabla 4. Caso 2. Relación de los objetivos del curso con el diseño de las vídeolecciones y la transferencia a la práctica docente.

\begin{tabular}{|c|c|c|c|}
\hline Curso & Objetivos del curso. & $\begin{array}{l}\text { Diseño de las vídeo- } \\
\text { lecciones }\end{array}$ & $\begin{array}{l}\text { Transferencia a la práctica } \\
\text { docente }\end{array}$ \\
\hline $\begin{array}{l}\text { Pasos básicos } \\
\text { para un } \\
\text { aprendizaje } \\
\text { personalizado } \\
\text { en el aula. }\end{array}$ & $\begin{array}{l}\text { Identificar y relacionar } \\
\text { componentes } \\
\text { características del } \\
\text { aprendizaje personalizado. } \\
\text { Orientar en la aplicación de } \\
\text { metodologías activas en el } \\
\text { proceso de aprendizaje que } \\
\text { permitan la personalización } \\
\text { del mismo, la participación } \\
\text { activa del alumnado y el } \\
\text { incremento de su } \\
\text { motivación. }\end{array}$ & $\begin{array}{l}\text { Se observa un estilo } \\
\text { dialógico de las clases y } \\
\text { carencias de tipo } \\
\text { narrativo, que sin duda, } \\
\text { podrían enriquecer este } \\
\text { ambiente r de } \\
\text { aprendizaje y servir de } \\
\text { ejemplo a otros } \\
\text { docentes sobre la } \\
\text { organización } \\
\text { contenido de } \\
\text { especializado. }\end{array}$ & $\begin{array}{l}\text { No se evidencian usos y } \\
\text { ejemplos concretos de } \\
\text { innovación pedagógica en } \\
\text { sus secuencias. Conviene } \\
\text { que se realicen video- casos } \\
\text { con ejemplos concretos de } \\
\text { personalización del } \\
\text { aprendizaje y se compartan } \\
\text { experiencias reales que } \\
\text { pueden utilizarse a manera } \\
\text { de serie con varias entregas. }\end{array}$ \\
\hline
\end{tabular}

Fuente: Elaboración propia

Tabla 5. Caso 3. Relación de los objetivos del curso con el diseño de las vídeolecciones y la transferencia a la práctica docente.

\begin{tabular}{|c|c|c|c|}
\hline Curso & Objetivos del curso. & $\begin{array}{l}\text { Diseño de las } \\
\text { vídeo-lecciones }\end{array}$ & $\begin{array}{l}\text { Transferencia a la práctica } \\
\text { docente }\end{array}$ \\
\hline $\begin{array}{l}\text { Ética en la } \\
\text { investigación } \\
\text { universitaria } \\
\left(5 .^{a} \text { edición) }\right.\end{array}$ & $\begin{array}{l}\text { El curso muestra y ayuda a entender } \\
\text { los aspectos que han de considerarse } \\
\text { para desarrollar investigaciones en la } \\
\text { Universidad de forma metodológica, } \\
\text { ética y legalmente aceptables. } \\
\text { Se explicarán aspectos éticos } \\
\text { generales comunes a toda } \\
\text { investigación y se tratarán } \\
\text { específicamente aquellos propios de } \\
\text { investigadores que se realizan con: a) } \\
\text { seres humanos (sus muestras y sus } \\
\text { datos personales), b) animales y c) } \\
\text { agentes biológicos y organismos } \\
\text { modificados genéticamente. }\end{array}$ & $\begin{array}{l}\text { Clases } \\
\text { magistrales con } \\
\text { ayuda del power } \\
\text { point } \\
\text { explicaciones } \\
\text { estándar. }\end{array}$ & $\begin{array}{l}\text { En este tipo de contenido se } \\
\text { evidencia la ausencia de } \\
\text { estrategias que puedan ser } \\
\text { transferibles al aula de clase } \\
\text { desde la perspectiva de la } \\
\text { construcción de ambientes de } \\
\text { aprendizaje y no desde la } \\
\text { transmisión de contenidos. }\end{array}$ \\
\hline
\end{tabular}

Fuente: Elaboración propia.

Cabe aclarar que los cursos presentan objetivos con verbos como explicar, orientar e identificar. Esto no necesariamente explica por qué las vídeo-lecciones son de carácter expositivo, pero si ayuda a entender que este tipo de cursos también tiene un alcance.

En general el estudiante no se encuentra con retos narrativos que causen impacto, curiosidad, diversión o empatía con el contenido. Se imprime un carácter plano y rígido 
y no se expresan elementos cotidianos de la personalidad del profesor, sino que se establece un ritmo acartonado y básico que desperdicia todo potencial audiovisual

\section{Discusión:}

Los hallazgos permiten establecer nuevas líneas de trabajo para la formación pedagógica en educación superior en la modalidad virtual. Al realizar un curso en formación pedagógica, los docentes esperarían poder conocer y aprender sobre modelos y estrategias de aprendizaje activo para incorporar en sus aulas. Esto supone un reto mayor para los diseñadores instruccionales de MOOC porque no sería razonable mostrar a los docentes un modelo tradicional de enseñanza. Sin embargo, como se ha analizado antes, los MOOC no cuentan con diseños instruccionales particulares según la población y sus necesidades de aprendizaje. Tal como lo menciona Area (2000) el uso de tecnología en la educación superior no ha impactado aun radicalmente los métodos tradicionales de enseñanza. En este sentido, dentro de lo que Salinas (2004) menciona como ideales dentro del diseño instruccional de este tipo de ambiente virtual, los recursos audiovisuales didácticos cobran especial relevancia toda vez que implica que cumplan un rol de mediador en el proceso de enseñanza - aprendizaje.

En cuanto al enfoque transmisionista de las clases analizadas, este tipo de diseño instruccional se convierte en una paradoja, porque si bien es cierto que un curso masivo ofrece autonomía, diversidad, e interactividad, cuanto más autónomo, diverso y abierto sea el curso, mayor es la posibilidad de limitar los aprendizajes por la falta de estructura y apoyo que tiende a ocurrir en la atención a tantas personas (Gikandi, 2011). Existe una brecha entre el discurso sobre los MOOC, la literatura sobre el deber ser de la formación pedagógica, las entidades académicas, los estudiantes, los diseñadores instruccionales y los maestros, con expectativas poco realistas sobre lo que significa enseñar y aprender a gran escala (Mackness, Mak y Williams, 2010; Ross, Sinclair, Knox, Bayne y Macleo, 2014).

Sin embargo, los riesgos metodológicos que se puedan plantear para un MOOC implican ajustes en el modelo de negocio, ya que este tipo de cursos especializados necesitan un diseño instruccional particular y adaptable que resulta costoso en términos de producción audiovisual. Sin embargo, diseños instruccionales distintos, novedosos y arriesgados, acompañados de un buen modelo de investigación, puede arrojar datos interesantes sobre el potencial de un curso masivo en línea para la formación de docentes.

Se espera que este tipo de análisis pueda aportar a la construcción de material audiovisual para cursos en línea proponiendo el uso de recursos audiovisuales de gran impacto a nivel narrativo. Los debates sobre la idoneidad de los MOOC para completar metas de aprendizaje aumentan en la medida en que se multiplican los cursos, lo que supone hacer un alto en el camino, superar expectativas y plantear propuestas ajustadas a las necesidades reales de formación en un sistema de educación globalizado, que si bien, no válida aún de manera formal un MOOC, paradójicamente sí ha entrado a producirlos en masa. Se enseña y se aprende diferente en línea, con lo cual, si queremos proponer cursos que apoyen la innovación pedagógica, el reto es poder modelar las transformaciones en formatos que propongan una profunda renovación de las metodologías de enseñanza - aprendizaje. 


\section{REFERENCIAS}

Allen, K. (2015). Que muestra el último estudio sobre los MOOC's? Revista de Educación virtual. Recuperado de https://bit.ly/1GrPIQO

Area, M. (2000). ¿Qué aporta internet al cambio pedagógico en la educación superior? En R. Pérez (Coord): Redes multimedia y diseños virtuales. Congreso Internacional de Comunicación, Tecnología y Educación llevado a cabo en la Universidad de Oviedo.

Bransford, J., Brown, A., y Cocking, R. (Eds.). (2000). How people learn: Brain, mind, experience, and school. Estados Unidos: National Academy Press.

Calvo, M., Rodríguez, C., y Fernández, E. (2016) ¿Cómo son los MOOC sobre educación? un análisis del cursos de temáticas pedagógica que se ofertan en castellano. Digital Education Review. Recuperado de https://bit.ly/2LbBtUI

Cáceres, M., Lara, L., Iglesias, C., García, R., Bravo, G., Cañedo, C., y Valdés, O. (2013). La formación pedagógica de los profesores universitarios. Una propuesta en el proceso de profesionalización del docente. Revista Iberoamericana De Educación, 33(1), 1-15. Recuperado de https://bit.ly/2Knm8EH

Goel, A., y Chauhan, J. (2015). An Analysis of Video Lecture in MOOC. CEUR Workshop Proceedings. 1356. Charalambos, V. (2000). Constructivism versus objectivism: implications for interaction, course design, and evaluation in distance education. International Journal of Educational Telecommunications, 6(4), 339-62.

Charalambos, V., y Glass, G.(2007). Teacher Professional Development and ICT: Strategies and Models. Yearbook of the National Society for the Study of Education. doi: 106. 87 102. 10.1111/j.1744-7984.2007.00116.x

Chehaybar, E. (2006). La percepción que tienen los profesores de educación media y superior y superior sobre su formación y su práctica docente. Revista Latinoamericana de Estudios Educativos, 219-259.

Dabner, N., Davis, N., y Zaka, P. (2012). Authentic project-based design of professional development for teachers studying online and blended teaching. Contemporary Issues in Technology and Teacher Education, 12 (1). Recuperado de http://bit.ly/2h3pLka

Dede, C. (2004). Preface. In C. Charalambos y G. V. Glass (Eds.), Online professional development for teachers. Greenwhich, CT: Information Age Publishing.

Gikandi, J. (2011). Achieving meaningful online learning through effective formative assessment. En G.Williams, P. Statham, N. Brown, B. Cleland (Eds.), Changing Demands, Changing Directions, 452-454. Proceedings ascilite Hobart.

Glance, D., Forsey, M. y Riley, M. (2013). The pedagogical foundations of massive open online courses. First Monday, 18 (5). doi: $10.5210 / \mathrm{fm} . v 18 i 5.4350$

González, R. (2005). La formación de los profesores de ingeniería. En: González Tirados y otros (eds.) Formación del Ingeniero, Objetivos, Métodos y Estrategias. Madrid: ICE Universidad Politécnica de Madrid, 419-443.
Ho, A., Chuang, I., Reich, J., Cody, A., Whitehill, J., Northcutt, C., Williams, J., Hansen, J., Lopez, G., y Petersen, R. (2015). HarvardX and MITx: Two Years of Open Online Courses. Working paper, doi: http://dx.doi.org/10.2139/ssrn.2586847

Igartua, J.(2006). Métodos cuantitativos de investigación en comunicación. Barcelona: Bosch.

Jobe, W., Öslund, C., y Svensson, L. (Enero, 2014). MOOCs for Professional Teacher Development. En SITE Society for Information Technology and Teacher Education International Conference. Congreso. Washington D.C. Recuperado de https://bit.ly/2ILN6qU

Krippendorff, K. (2013). Content Analysis: An Introduction to Its Methodology. 2nd Edition. SAGE Publications. 51

Lambert, C. (2012). Twilight of the lecture. En: Harvard Magazine. Recuperado de https://bit.ly/2NdULe6

Lee, V. (2010). Program Types and Protypes. En K. Gillespie, D. Robertson., y W. Bergguist (Eds.), Guide to Faculty Development, Second Edition. (pp. 21-34). Jossey Bass.

Lei, C., Yeung, Y., Kwok, T., Lau, R., y Ang, A. (2016) "Leveraging videos and forums for small-class learning experience in a MOOC environment," 2016 IEEE International Conference on Teaching, Assessment, and Learning for Engineering (TALE), Bangkok, 409-411.

León, J., y Correa, E. (2011). La imagen y su papel en la narrativa audiovisual. Universidad de Medellín. Imago. Recuperado de https://bit.ly/1HcPzAp

Literat, I. (2015). Implications of massive open online courses for higher education: mitigating or reifying educational inequities? Higher Education Research y Development, 34 (6), 11641177.

Lovegrove, E., Mackness, J., Roberts G., y Waite, M. (2013). Liminal Participants and Skilled Orienteers: Learner Participation in a MOOC for New Lecturers. Merlot Journal of Online Learning and Teaching 9 (2). Recuperado de https://bit.ly/2tHNTNX

Lowenthal, P. (2008). Online Faculty Development and Storytelling: an Unlikely Solution to improving teacher quality. Merlot Journal of Online Learning and Teaching, 4 (3), 349356. Recuperado de https://bit.ly/2KBoLib

Martínez, A., y De Castro, A. (2012). Transformar para educar. Barranquilla, Colombia: Editorial Universidad del Norte

Mackness, J., Mak, S., y Williams, R. (2010). The ideals and reality of participating in a MOOC. Proceedings of the 7th International Conference on Networked Learning, 266-274.

Merrill, D. (2002). First Principles of Instruction. ETRyD 50, (3),. 43-59.

Monney, K. (2010). Working with a Faculty Development Committee. En K. Gillespie, D. Robertson y W. Bergguist (Eds.), Guide to Faculty Development, Second Edition, 53-6 
Montiel, G. (2009). Formación docente a distancia en línea. Un modelo desde la matemática educativa. Innovación Educativa, 9 (46), 89-95.

Moscoso, F. y Hernández, A. (2015). La formación pedagógica del docente universitario: contemporáneo. Revista

un reto del mundo
Cubana de Educación
Superior, 34(3), 140-154.

Padilla, A., López., M y Rodríguez, A. (2015). La formación del docente universitario. Concepciones teóricas y metodológicas.. Revista Universidad y Sociedad, 7(1), 86-90.

Rama, C. (2008). Tipología de las tendencias de la virtualización de la educación superior en américa latina. Revista diálogo educacional, 8 (24), 341-355.

Rangel, A. (2007). Aportaciones metodológicas para el análisis de la práctica pedagógica a través del video. En Casanova (Presidencia). XI Congreso Nacional de Investigación Educativa. Conferencia llevada a cabo en la UNAM, México.

Raposo, M., Martínez, E., y Sarmiento, J. (2015). Un estudio sobre los componentes pedagógicos de los cursos online masivos. Comunicar, 22 (44), 27-35.

Rose, K. (2009). Student Perceptions of the Use of InstructorMade Videos in Online and Face-to-Face Classes. MERLOT Journal of Online Learning and Teaching, 5 (3). Recuperado de http://bit.ly/2IGAPwL

Ross, J., Sinclair, C., Knox, J., Bayne, N., y Macleod, H. (2014). Teacher experiences and academic identity: The missing components of MOOC pedagogy. Journal of Online Learning and Teaching, 10 (1), 57-69. Recuperado de http://bit.ly/2NdWMqG

Sampieri, R., Fernández, C., y Baptista, M. (2014). Metodología de la investigación (6ta edición) México : McGraw Hill.

Silva, I., y Salgado, I. (2014). Utilización de MOOCs en la formación docente: ventajas, desventajas y peligros. Profesorado Revista de curriculum y formación del profesorado, 18 (1), 155-166. Recuperado de http://bit.ly/2Nb1HZr

Stevens, E., Dunlap, J., Bates, B., Lowenthal, P., Wray, M., y Switzer, T. (2005). Faculty development attitudes and motivators. Paper presented at the annual meeting of the Northern Rocky Mountain Educational Research Association, Jackson, WY

Singh, M. (2014). The Potential of the UNESCO Guidelines for the Recognition, Validation and Accreditation of the Outcomes of Non-Formal and Informal Learning for Continuing Higher Education in Latin America". En Betti, Maurizio; Kachele Mónica; Mousalli, Gloria y Rivas, Francklin. Aprendizaje a lo largo de la vida: Realidades, Desafíos y Oportunidades de la Educación Superior en América Latina. Chile, 14 - 47.

Shulman, L. (1986). Those who understand: knowledge growth in teaching. Educational Researcher, 15(2), 4-14.

Vivian, R., Falkner, K., y Falkner, N. (2014). Addressing the challenges of a new digital technologies curriculum: MOOCs as a scalable solution for teacher professional development. Research In Learning Technology 22 (1). Recuperado de http://bit.ly/2IJVyX
Villalobos, A., y Melo, Y. (2008). La formación del profesor universitario: Aportes para su discusión. Recuperado de http://bit.ly/2tJkcvE

Winslett, G. (2014). What Counts as Educational Video?: Working toward Best Practice Alignment between Video Production Approaches and Outcomes. Australasian Journal of Educational Technology, 30(5), 487-502. 